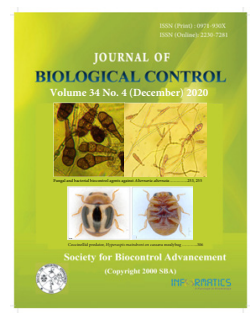

\title{
Management of white grub, Holotrichia consanguinea (Blanchard) using biocontrol agents in sugarcane in coastal Andhra Pradesh
}

\author{
B. RAMANUJAM ${ }^{1 *}$, M. VISALAKSHI ${ }^{2}$, JAGADEESH PATIL ${ }^{1}$ and B. POORNESHA ${ }^{1}$ \\ ${ }^{1}$ ICAR-National Bureau of Agricultural Insect Resources, H.A. Farm Post, Bellary Road, Hebbal, Bengaluru, Karnataka, India \\ ${ }^{2}$ Regional Agricultural Research Station, ANGRAU, Anakapalli, Andhra Pradesh, India \\ *Corresponding author E-mail: bonamramanujam58@gmail.com
}

\begin{abstract}
White grubs are one of the major pests of sugarcane crop in coastal Andhra Pradesh, India. The present study was taken up to assess the efficacy of biocontrol agents viz., entomopathogenic fungi and entomopathogenic nematodes for management of white grub, Holotrichia consanguinea in sugarcane ecosystem during 2015-16 and 2016-17. Two entomopathogenic fungi, Metarhizium anisopliae (NBAIR Ma4 strain) and Beauveria bassiana (NBAIR Bb5a), two entomopathogenic nematodes (Heterorhabditis indica NBAIR-H38 and Steinernema carpocapsae NBAII Sc 05), Neem cake, Phorate 10G were evaluated through soil application as different treatments in white grub infested sugarcane field in coastal Andhra Pradesh for two seasons during 2015 and 2016-17. All biocontrol agent treatments were better than the insecticidal/neem cake application in the management of sugarcane white grubs.However, based on cost benefit ratio of the treatments, soil application of M. anisopliae @ $2.5 \mathrm{~kg}$ along with $250 \mathrm{~kg}$ farm yard manure per hectare (twice application in July and August) proved superior to other biocontrol agent treatments. Soil application of biocontrol agents, Phorate and Neem cake did not show anydeleterious effect on non-target organisms like, earthworms, earwigs, spiders, coccinellids and chrysopids.
\end{abstract}

KEY WORDS: Biocontrol agents, Holotrichia consanguinea, sugarcane, white grubs

(Article chronicle: Received: 16-11-2020; Revised: 28-12-2020; Accepted: 30-12-2020)

\section{INTRODUCTION}

Sugarcane is an important cash crop grown in India and India is second largest producer of sugarcane in the world. In Andhra Pradesh, sugarcane is grown in 2.40 lakh hectares and 136 lakh tons of sugarcane is produced in the state. It is largely grown in Visakhapatnam, West Godavari, East Godavari, Chittoor, Krishna, Vizayanagaram, Srikakulam and Nellore Districts with 90 per cent of the area under this crop (Kumar and Suneetha, 2016). White grub, Holotrichia consanguinea, Blanchard (Coleoptera: Scarabaeidae) is an important soil pest in sugarcane crop in India. It causes severe damage to commercial crops in India. In recent years, white grubs extending its spatial range due to monoculture of sugarcane and minimal varietal diversity. It has affected the sugarcane crop in certain parts of Andhra Pradesh. The adult beetles feed on foliage of host trees like neem, acacia etc., late larval stages feed on roots of sugarcane and underground portions of stalks. White grub infestation on roots results in yellowing of leaves, wilting and drying of clumps. Infested canes lose weight, dry up, collapse and become unfit for crushing. White grub damage generally occurs in patches leaving gaps and severe infestation results in devastation of entire field. Severe symptoms of damage by older grubs generally observed late in the season resulting in complete loss of crop as curative chemical control is ineffective against late stage grubs. The yield loss due to white grubs was reported to be as high as 100 per cent in Tamil Nadu (Selvi et al., 2010). Several practices were adopted for the management of white grubs including cultural, mechanical, biological, chemical and integrated methods (Sahayaraj and Borgio, 2009; Srikanth and Singaravelu, 2011). Pest management strategy depends primarily on the use of highly poisonous poor graded chemical pesticides. Chemical control is practically uneconomical, difficult and associated with high cost, environmental pollution and pesticide residues. Hence, there is need for effective ecofriendly and economically feasible strategy for the control of white grubs in sugarcane. Entomofungal Pathogens (EPF) like Beauveria bassiana, Metarhizium anisopliae, Entomopathogenic Nematodes (EPN) like, Heterorhabditis sp. and Steinernema sp. were reported to be cost effective and eco-friendly management of white grubs pests in various crops. These biocontrol agents are also self-perpetuating in nature. Hence, studies on field evaluation of EPF and EPN were carried out for the 
Management of white grub, Holotrichia consanguinea using biocontrol agents in sugarcane

management of white grubs in sugarcane in an endemic area of Andhra Pradesh.

\section{MATERIALS AND METHODS}

\section{Field evaluation}

The field trials were conducted during 2015-16 \& 2016-17against white grubs in sugarcane field (variety, 2003V46) at RARS, Anakapalli, Andhra Pradesh, India (Latitude-17.6896 ${ }^{\circ} \mathrm{N}$ and Longitude-83.0024 ${ }^{\circ} \mathrm{E}$ ). The field trials were laid out with nine treatments as given below in Randomized Block Design (RDB) in 5 ha area. Each treatment had three replications distributed randomly in 0.555 hectare area. First year sugarcane was ratoon crop which was started in January 2015 and was harvested in December 2015. Second year crop was fresh planting sown in March 2016 and was harvested in January 2017. All the agronomic practices with recommended dose of fertilizers were followed till the crop harvest to maintain good plant health as per the package of practices of sugarcane crop (Anon 2018).

\section{Treatments : 9}

T1: Metarhizium anisopliae (NBAIR Ma4) 2.0\% W.P. talc formulation@2.5 kg ha-1 $1 \times 10^{8} \mathrm{Cfu} / \mathrm{g}$ (minimum) in $250 \mathrm{~kg}$ FYM (2 times soil application, July and August)

T2: Beauveria bassiana (NBAIR Bb5a) 2.0\% W.P. talc formulation@2.5 $\mathrm{kg} \mathrm{ha}^{-1} 1$ x10 $10^{8} \mathrm{Cfu} / \mathrm{g}$ in $250 \mathrm{~kg}$ FYM (2 times soil application, July and August)

T3: Heterorhabditis indica (NBAIR H38) WP @, 20 kg $\mathrm{ha}^{-1}$ in $150 \mathrm{~kg}$ moist sand ha ${ }^{-1}$ (Single soil application in July)

T4: Heterorhabditis indica (NBAIR H38)WP@20 kg $\mathrm{ha}^{-1}$ in $150 \mathrm{~kg}$ moist sand ha-1 soil application two times at two month interval(July and September)

T5: Steinernema carpocapsae (NBAII Sc05) WP@20 $\mathrm{kg} \mathrm{ha}^{-1}$ in $150 \mathrm{~kg}$ moist sand ha-(Single soil application in July)

T6: Steinernema carpocapsae (NBAII Sc05) WP@20 $\mathrm{kg} \mathrm{ha}^{-1}$ in $150 \mathrm{~kg}$ moist sand $\mathrm{ha}^{-1}$ soil application two times at two month interval(July and September)

T7: Neem cake@ 900 kg ha-1(Single soil application in July)

T8: Phorate10G@15kg ha-1(Single soil application in July)

T9: Untreated control 250kg of FYM without enrichment of fungus (2 times application, July and August)

\section{Preparation of talc formulation (2\% WP) of $M$. anisopli- ae and $B$. bassiana}

The fungi, $M$. anisopliae NBAIR-Ma4and B. bassiana NBAIR Bb-5a were grown in 1 litre conical flask (containing $500 \mathrm{ml}$ medium) of Sabouraud's Dextrose Yeast extract Broth (SDYB) (Dextrose $20 \mathrm{~g}$, Mycological peptone $10 \mathrm{~g}$, yeast extract $5 \mathrm{~g}$ in $1 \mathrm{~L}$ of distilled water)in orbital shaker at $25 \pm$ $2{ }^{\circ} \mathrm{C}$ temperature, $150 \mathrm{rpm}$ for 8 days. The 8 days old culture broth was centrifuged at 5000rpm for 10mins, supernatant was discarded and the pellet was mixed in sterilized talcum powder at $2 \%$ (20grams of pellet in $1 \mathrm{~kg}$ talc) in sterilized tray under laminar air flow and dried to $8 \%$ moisture. The talc formulations of $M$. anisopliae NBAIR-Ma4 and $B$. bassiana NBAIR Bb-5a contained $1.8 \times 10^{8}$ and $1.0 \times 10^{8} \mathrm{cfu} / \mathrm{g}$ respectively. The formulations were stored in milky white polypropylene pouches for further use in field evaluation trials.

\section{Preparation of $M$. anisopliae/B. bassiana enriched farm- yard manure}

$2.5 \mathrm{~kg}$ of talc formulation ( $2 \% \mathrm{WP})$ of each fungus was mixed thoroughly with $250 \mathrm{~kg}$ FYM in shaded area in the field and incubated for 15 days with intermittent sprinkling of water. After 15 days, the fungus enriched Farmyard manure was used for soil application.

\section{Application of treatments}

In T1 and T2 treatments, soil application of $M$. anisopliae/B. bassiana enriched Farmyard manure@,250 kg/ ha was done two times in the furrows adjoining the root zone of sugar cane clumps. The first application was in July and the second application was done in August after one month of first application in each year. In T3 and T5 treatments, $H$. indica/S. carpocapsae were applied to the soil@20 kg ha-1 in $150 \mathrm{~kg}$ moist sand ha-1 in July in each year. In T4 and T6 treatments, $H$. indica/S. carpocapsae were applied to the soil two times at two months intervals@20 kg ha ${ }^{-1}$ in $150 \mathrm{~kg}$ moist sand ha ${ }^{-1}$ in July and September in each year. In case of T7 treatment, soil application of Neem cake @ 500kg/ha and in T8 treatment, Phorate10 G@15 kg/ha was done in July each year. In T9 treatment, soil application of Farmyard manure (without fungus enrichment)@250 kg/ha was done similarly two times in July and August in each year.

\section{Observations}

\section{Effect on plant damage, white grub population and crop yield}

Observations on number of plants damaged by white grubs $(\%)$, number of white grubs per 10 meter row were recorded at monthly interval till the crop was harvested (July- 
RAMANUJAM et al.

December 2015during 2015 crop season \& July 2016-January 2017 during 2016-17 crop season). Yield was recorded in December 2015 in the first year of trial and in January 2017 in second year trial and expressed as tons per hectare. Monthly data on plant damage, white grub population and cane yield were analyzed statistically for drawing inferences. The cost benefit ratio of the treatments has been calculated based on the formula given below.

BC Ratio $=\mathrm{NR} / \mathrm{CC}$; NR- Net Returns; CC- Cost of Cultivation

\section{Effect on non-target organisms}

Non target organisms which were inhabiting soil viz., earthworms and earwigs were recorded in a cubic meter area by removing the soil a day before treatment imposition and after 30 days of treatment. The predators like spiders, coccinellids and chrysopids per plant were recorded in each treatment a day before treatment imposition and after 30 days of treatment which were averaged and subjected to statistical analysis.

\section{RESULTS AND DISCUSSION}

The experimental results on the efficacy of different treatments are presented in Table-1 (2015), Table-2 (2016-17) and Table-3 (Pooled analysed data for 2 years). The results on the effect of different treatments on Non-Target organisms are presented in Table-4 (2015) and Table-5 (2016-17)

\section{Plant damage due to white grubs (\%)}

During the first year field trial (2015), the per cent plants damaged by white grubs was lowest $(1.47 \%)$ in $M$. anisopliae (Strain no. NBAIR Ma4) 2.0\% W.P. Formulation@ @2.5kgha${ }^{1}$ (twice application) treated plots which was statistically at par with other treatments like, T4: H. indica (NBAIR H38) WP @ 20kg ha-1 (twice application) with plant damage of 2.17\%, T6 and T5: S. carpocapsae (NBAII Sc05) WP @ $20 \mathrm{~kg} \mathrm{ha}^{-1}$ (twice and single application) with damage of 2.79 and $3.16 \%$ and T2: B. bassiana (NBAIR-Bb5a) 2.0\% W.P. Formulation@ $2.5 \mathrm{~kg} \mathrm{ha}^{-1}$ (twice application)with 3.0\% plant damage (Table 1). Other treatments like, Phorate 10G/Neem cake application/untreated control recorded significantly very high plant damage of 12.65, 25.0 and $48.0 \%$ (Table 1).

During the second year field trial (2016-17), the lowest per cent of plants damaged by white grubs was observed in $\mathrm{T} 4$ treatment(1.6\%), H. indica (NBAIR H38)WP @ $20 \mathrm{~kg} \mathrm{ha}^{-1}$ (twice application) which was statistically at par with other treatments like, T3, H. indica (NBAIR H38)WP @ $20 \mathrm{~kg}$ $\mathrm{ha}^{-1}$ (single application) with plant damage of $2.81 \%, \mathrm{~T} 1, M$. anisopliae (NBAIR Ma4)@2.5 $\mathrm{kgha}^{-1}$ (twice application) with plant damage of $4.04 \%$, T6 and T5: S. carpocapsae (NBAII Sc05)WP@ $20 \mathrm{~kg} \mathrm{ha}^{-1}$ (twice and single application) with plant damage of 4.58 and $4.12 \%$ and T2: B. bassiana (NBAIR Bb5a)@2.5 $\mathrm{kgha}^{-1}$ (twice application) with 5.12\% plant damage (Table 1). Other treatments like, Phorate 10G/Neem cake application/untreated control recorded significantly high plant damage of 9.17, 11.29 and $16.41 \%$ (Table 2).

The pooled data analysis of two years indicates that all biocontrol agent treatments (T1, T2, T3, T4, T5 and T6 treatments were at par with each other with regard to percent plant damage (2.75-4.06\%), although T1 (M. anisopliae) and T4 (H. indica NBAIR H38) treatments showed lowest percent plant damage (2.275 and 2.99\%) (Table 3).

\section{Incidence of white grub population}

During 2015 trial, the white grub population was significantly low $(0.67$ grubs/ $10 \mathrm{~m}$ row $)$ in $M$. anisopliae (Strain no. NBAIR Ma4) 2.0\% W.P. Formulation @ 2.5 kgha${ }^{1}$ (twice application) treated plots and superior to all other treatments (T2, T3, T4, T5, T6, T7 and T8) which showed white grub population in the range of 1.33 to 9.00 grubs $/ 10 \mathrm{~m}$ row. Untreated control recorded significantly very high grub population 13.67 grubs/10 $\mathrm{m}$ row (Table 1).

In 2016-17 trial, the lowest white grub population was recorded in T4 treatment (1.46grubs $/ 10 \mathrm{~m}$ row), $H$. indica (NBAIR H38) (twice application) which was statistically at par with other treatments like, T3, H. indica (NBAIR H38) (single application) with 2.67 grubs $/ 10 \mathrm{~m}, \mathrm{~T} 2, M$. anisopliae treatment with 2.67 grubs/10m, T5: S. carpocapsae (NBAII Sc05) (single application) with 3.62 grubs/10mand T2, $B$. bassiana with 4.08grubs/10 $\mathrm{m}$ and T6 S. carpocapsae (NBAII $\mathrm{Sc} 05$ ) (twice application) with 4.31 grubs $/ 10 \mathrm{~m}$ row (Table 1 ). Other treatments like, Phorate 10G/Neem cake application/ untreated control recorded significantly high grub population of 9.12, 7.01 and 13.82 grubs/10m row (Table 2).

The pooled data analysis of two years indicates that all biocontrol agent treatments (T1, T2, T3, T4, T5 and T6 treatments were at par with each other with regard white grub population(1.40-2.99 grubs/10m row), although T4 (H. indica NBAIR H38) treatment showed lowest grub population of 1.4 grubs/10m row (Table-3).

\section{Cane yield}

In the first year trial (2015), the cane yield was significantly high $(87.41 \mathrm{t} / \mathrm{ha})$ in $M$. anisopliae (NBAIRMa4) 2.0\% W.P. Formulation@2.5 $\mathrm{kgha}^{-1}$ (twice application) treated plots and superior to all other treatments (T2, T3, T4, $\mathrm{T} 5, \mathrm{~T} 6, \mathrm{~T} 7$ and T8) which recorded cane yield in the range of 
Table 1. Efficacy of bioagents against white grubs in sugarcane crop during 2015-16

\begin{tabular}{|c|c|c|c|c|c|c|c|}
\hline Treatment & $\begin{array}{l}\text { Per cent plants } \\
\text { damaged by } \\
\text { white grubs }\end{array}$ & $\begin{array}{l}\text { Per cent reduc- } \\
\text { tion in plant } \\
\text { damage by } \\
\text { white grubs } \\
\text { over control } \\
\end{array}$ & $\begin{array}{l}\text { White } \\
\text { grubs/ } \\
\text { 10m row }\end{array}$ & $\begin{array}{l}\text { Per cent } \\
\text { reduction in } \\
\text { white grub } \\
\text { population } \\
\text { over control }\end{array}$ & $\begin{array}{l}\text { Cane } \\
\text { yield } \\
\text { t/ha }\end{array}$ & $\begin{array}{l}\text { Yield } \\
\text { increase over } \\
\text { control } \\
(\%)\end{array}$ & $\begin{array}{l}\mathrm{C}: \mathrm{B} \\
\text { ratio }\end{array}$ \\
\hline $\begin{array}{l}\text { T1: Metarhizium anisopliae } \\
\text { (NBAIR Ma4) 2.0\% W.P. @ } \\
2.5 \mathrm{~kg} \mathrm{ha}^{-1} \\
1 \times 10^{8} \mathrm{Cfu} / \mathrm{g} \text { (min.) in } 250 \mathrm{~kg} \\
\text { FYM (2 times application) }\end{array}$ & $1.47^{\mathrm{a}}$ & 96.94 & $0.67^{\mathrm{a}}$ & 95.10 & $87.41^{\mathrm{a}}$ & 71.48 & 2.0 \\
\hline $\begin{array}{l}\text { T2: Beauveria bassiana } \\
\text { (NBAIR Bb5a) } 2.0 \% \text { W.P. @ } \\
2.5 \mathrm{~kg} \mathrm{ha}^{-1} \\
1 \times 10^{8} \mathrm{Cfu} / \mathrm{g} \text { in } 250 \mathrm{~kg} \mathrm{FYM} \mathrm{(2} \\
\text { times application) }\end{array}$ & $3.0^{\mathrm{a}}$ & 93.76 & $1.67^{b}$ & 87.78 & $74.99^{\mathrm{b}}$ & 66.76 & 1.71 \\
\hline $\begin{array}{l}\text { T3: Heterorhabditis indica } \\
\text { (NBAIR H38)WP @ } 20 \mathrm{~kg} \\
\text { ha }^{-1} \text { in } 150 \mathrm{~kg} \text { moist sand ha }{ }^{-1}\end{array}$ & $4.61^{\mathrm{ab}}$ & 90.40 & $2.67^{\mathrm{c}}$ & 80.47 & $70.77^{\mathrm{c}}$ & 64.77 & 1.49 \\
\hline 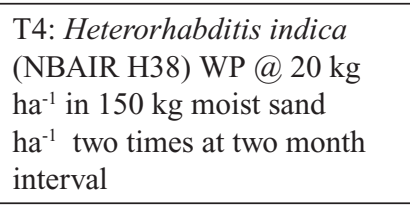 & $2.17^{\mathrm{a}}$ & 95.48 & $1.33^{\mathrm{b}}$ & 90.27 & $77.08^{b}$ & 67.66 & 1.50 \\
\hline $\begin{array}{l}\text { T5: Steinernema carpocapsae } \\
\text { (NBAII Sc05)WP @ } 20 \mathrm{~kg} \\
\mathrm{ha}^{-1} \text { in } 150 \mathrm{~kg} \text { moist sand ha }\end{array}$ & $3.16^{\mathrm{a}}$ & 93.42 & $1.67^{b}$ & 87.78 & $70.09^{c}$ & 64.43 & 1.48 \\
\hline $\begin{array}{l}\text { T6: Steinernema carpocapsae } \\
\text { (NBAII Sc05)WP @ } 20 \mathrm{~kg} \\
\text { ha }^{-1} \text { in } 150 \mathrm{~kg} \text { moist sand ha }{ }^{-1} \\
\text { two times attwomonths interval }\end{array}$ & $2.79^{\mathrm{a}}$ & 94.19 & $1.67^{\mathrm{b}}$ & 87.78 & $73.42^{\mathrm{bc}}$ & 66.04 & 1.43 \\
\hline T7: Neem cake@ 500 kg ha ${ }^{-1}$ & $25.00^{c}$ & 47.96 & $9.0^{\mathrm{e}}$ & 34.16 & $61.90^{\mathrm{d}}$ & 59.72 & 1.36 \\
\hline T8: Phorate 10G@15kg ha-1 & $12.65^{\mathrm{b}}$ & 73.67 & $6.33^{\mathrm{d}}$ & 53.69 & $61.59^{\mathrm{d}}$ & 59.52 & 1.30 \\
\hline $\begin{array}{l}\text { T9: Untreated control only } \\
\text { 250kg FYM }\end{array}$ & $48.04^{\mathrm{d}}$ & & $13.67^{\mathrm{f}}$ & - & $24.93^{\mathrm{e}}$ & & 0.57 \\
\hline $\mathrm{CD}(\mathrm{P}=0.05)$ & 8.35 & & 0.51 & & 3.70 & & \\
\hline $\mathrm{CV} \%$ & 21.23 & & 14.13 & & 2.42 & & \\
\hline
\end{tabular}

Values in column followed by different letter $(a, b, c)$ are significantly different from each other 
Table 2. Efficacy of bioagents against white grubs in sugarcane crop during 2016-17

\begin{tabular}{|c|c|c|c|c|c|c|c|}
\hline Treatment & $\begin{array}{l}\text { Per cent } \\
\text { plants } \\
\text { damaged by } \\
\text { white grubs }\end{array}$ & $\begin{array}{l}\text { Per cent re- } \\
\text { duction over } \\
\text { control }\end{array}$ & $\begin{array}{l}\text { White } \\
\text { grubs/ } \\
\text { 10m row }\end{array}$ & $\begin{array}{l}\text { Per cent } \\
\text { reduction in } \\
\text { white grub } \\
\text { population } \\
\text { over control }\end{array}$ & $\begin{array}{l}\text { Cane } \\
\text { yield } \\
\text { t/ha }\end{array}$ & $\begin{array}{l}\text { Yield } \\
\text { increase over } \\
\text { control } \\
(\%)\end{array}$ & $\begin{array}{l}\mathrm{C}: \mathrm{B} \\
\text { ratio }\end{array}$ \\
\hline $\begin{array}{l}\text { T1: Metarhizium anisopliae } \\
\text { (NBAIR Ma4) } 2.0 \% \text { W.P.@ } 2.5 \mathrm{~kg} \\
\text { ha }{ }^{-1} 1 \times 10^{8} \mathrm{Cfu} / \mathrm{g} \text { (min.) in } 250 \mathrm{~kg} \\
\text { FYM (2 times application) }\end{array}$ & $4.04^{\mathrm{ab}}$ & 75.38 & $2.96^{\mathrm{ab}}$ & 78.58 & $97.73^{\mathrm{a}}$ & 51.79 & 2.03 \\
\hline $\begin{array}{l}\text { T2: Beauveria bassiana (NBAIR } \\
\text { Bb5a) } 2.0 \% \text { W.P. @ } 2.5 \mathrm{~kg} \mathrm{ha}^{-1} \\
1 \times 10^{8} \mathrm{Cfu} / \mathrm{g} \text { in } 250 \mathrm{~kg} \text { FYM ( } 2 \\
\text { times application) }\end{array}$ & $5.12^{\mathrm{ab}}$ & 68.8 & $4.08^{\mathrm{ab}}$ & 70.48 & $92.82^{\mathrm{ab}}$ & 49.23 & 1.93 \\
\hline $\begin{array}{l}\text { T3: Heterorhabditis indica (NBAII } \\
\text { H38)WP @ } 20 \mathrm{~kg} \mathrm{ha}^{-1} \text { in } 150 \mathrm{~kg} \\
\text { moist sand ha }{ }^{-1}\end{array}$ & $2.81^{\mathrm{a}}$ & 82.88 & $2.67^{\mathrm{a}}$ & 80.68 & $99.8^{\mathrm{a}}$ & 52.78 & 1.93 \\
\hline $\begin{array}{l}\text { T4: Heterorhabditis indica (NBAII } \\
\mathrm{H} 38 \text { ) WP @ } 20 \mathrm{~kg} \mathrm{ha}^{-1} \text { in } 150 \mathrm{~kg} \\
\text { moist sand ha }{ }^{-1} \text { two times at two } \\
\text { months interval }\end{array}$ & $1.60^{\mathrm{a}}$ & 90.25 & $1.46^{\mathrm{a}}$ & 89.44 & $101.1^{\mathrm{a}}$ & 53.39 & 1.82 \\
\hline $\begin{array}{l}\text { T5: Steinernema carpocapsae } \\
\text { (NBAII Sc05) WP @ } 20 \mathrm{~kg} \mathrm{ha}^{-1} \text { in } \\
150 \mathrm{~kg} \text { moist sand ha }{ }^{-1}\end{array}$ & $4.12^{\mathrm{ab}}$ & 74.89 & $3.62^{\mathrm{ab}}$ & 73.81 & $98.49^{\mathrm{a}}$ & 52.16 & 1.90 \\
\hline $\begin{array}{l}\text { T6: Steinernema carpocapsae } \\
\text { (NBAII Sc05) WP @ } 20 \mathrm{~kg} \mathrm{ha}^{-1} \text { in } \\
150 \mathrm{~kg} \text { moist sand ha }{ }^{-1} \text { two times } \\
\text { attwo months interval }\end{array}$ & $4.58^{\mathrm{ab}}$ & 72.09 & $4.31^{\mathrm{ab}}$ & 68.81 & $98.70^{\mathrm{a}}$ & 52.26 & 1.77 \\
\hline T7: Neem cake@ @ 500 kg ha ${ }^{-1}$ & $11.29^{\mathrm{bc}}$ & 31.20 & $9.12^{\mathrm{b}}$ & 34.01 & $86.02^{\mathrm{b}}$ & 45.22 & 1.72 \\
\hline T8: Phorate 10G@15kg ha-1 & $9.17^{\mathrm{b}}$ & 44.12 & $7.01^{\mathrm{b}}$ & 49.28 & $69.74^{\mathrm{c}}$ & 32.43 & 1.45 \\
\hline $\begin{array}{l}\text { T9: Untreated control only } 250 \mathrm{~kg} \\
\text { FYM }\end{array}$ & $16.41^{\mathrm{c}}$ & & $13.82^{\mathrm{c}}$ & - & $47.12^{\mathrm{d}}$ & & 0.98 \\
\hline $\mathrm{CD}(\mathrm{P}=0.05)$ & 5.37 & & 4.33 & & 8.88 & & \\
\hline $\mathrm{CV} \%$ & 22.15 & & 19.51 & & 5.79 & & \\
\hline
\end{tabular}

Values in column followed by different letter $(a, b, c)$ are significantly different from each other 
Management of white grub, Holotrichia consanguinea using biocontrol agents in sugarcane

Table 3. Efficacy of bioagents against white grubs in sugarcane crop (Pooled data of 2015 and 2016-17)

\begin{tabular}{|c|c|c|c|c|c|c|c|}
\hline Treatment & $\begin{array}{l}\text { Per cent } \\
\text { plants dam- } \\
\text { aged by } \\
\text { white grubs }\end{array}$ & $\begin{array}{l}\text { Per cent } \\
\text { reduction in } \\
\text { plant damage } \\
\text { over control }\end{array}$ & $\begin{array}{l}\text { White } \\
\text { grubs/ } \\
\text { 10m row }\end{array}$ & $\begin{array}{l}\text { Per cent reduc- } \\
\text { tion in white } \\
\text { grub popu- } \\
\text { lation over } \\
\text { control }\end{array}$ & $\begin{array}{l}\text { Cane yield } \\
\text { t/ha }\end{array}$ & $\begin{array}{l}\text { Yield } \\
\text { increase } \\
\text { over } \\
\text { control(\%) }\end{array}$ & $\begin{array}{l}\mathrm{C}: \mathrm{B} \\
\text { ratio }\end{array}$ \\
\hline $\begin{array}{l}\text { T1: Metarhizium anisopliae } \\
\text { (NBAIR Ma 4) 2.0\% W.P.@ } 2.5 \mathrm{~kg} \\
\text { ha }^{-1} 1 \times 10^{8} \mathrm{Cfu} / \mathrm{g} \text { (min.) in } 250 \mathrm{~kg} \\
\text { FYM (2 times application) }\end{array}$ & $2.75^{\mathrm{a}}$ & 91.47 & $1.99^{\mathrm{a}}$ & 85.53 & $92.58^{\mathrm{a}}$ & 61.09 & 2.02 \\
\hline $\begin{array}{l}\text { T2: Beauveria bassiana (NBAIR } \\
\text { Bb5a) } 2.0 \% \text { W.P. @ } 2.5 \mathrm{~kg} \mathrm{ha}^{-1} 1 \\
\text { x } 10^{8} \mathrm{Cfu} / \mathrm{g} \text { in } 250 \mathrm{~kg} \text { FYM ( } 2 \text { times } \\
\text { application) }\end{array}$ & $4.06^{\mathrm{ab}}$ & 87.4 & $2.88^{\mathrm{a}}$ & 79.05 & $83.90^{\mathrm{b}}$ & 57.07 & 1.82 \\
\hline $\begin{array}{l}\text { T3: Heterorhabditis indica } \\
\text { (NBAIR H38)WP @ } 20 \mathrm{~kg} \mathrm{ha}^{-1} \text { in } \\
150 \mathrm{~kg} \text { moist sand ha }{ }^{-1}\end{array}$ & $3.71^{\mathrm{ab}}$ & 88.49 & $2.67^{\mathrm{a}}$ & 80.58 & $85.29^{\mathrm{ab}}$ & 57.77 & 1.71 \\
\hline $\begin{array}{l}\text { T4: Heterorhabditis indica } \\
\text { (NBAIR H38) WP @ } 20 \mathrm{~kg} \mathrm{ha}^{-1} \text { in } \\
150 \mathrm{~kg} \text { moist sand ha }{ }^{-1} \text { two times } \\
\text { at two months interval }\end{array}$ & $2.99^{\mathrm{a}}$ & 90.72 & $1.40^{\mathrm{a}}$ & 89.82 & $89.09^{\mathrm{ab}}$ & 59.57 & 1.69 \\
\hline $\begin{array}{l}\text { T5: Steinernema carpocapsae } \\
\text { (NBAII Sc05)WP @ } 20 \mathrm{~kg} \mathrm{ha}^{-1} \text { in } \\
150 \mathrm{~kg} \text { moist sand ha }\end{array}$ & $4.00^{\mathrm{ab}}$ & 87.58 & $2.65^{\mathrm{a}}$ & 80.73 & $84.29^{b}$ & 57.27 & 1.66 \\
\hline $\begin{array}{l}\text { T6: Steinernema carpocapsae } \\
\text { (NBAII Sc05) WP @ } 20 \mathrm{~kg} \mathrm{ha}^{-1} \text { in } \\
150 \mathrm{~kg} \text { moist sand ha }{ }^{-1} \text { two times } \\
\text { at two months interval }\end{array}$ & $3.69^{\mathrm{ab}}$ & 88.55 & $2.99^{\mathrm{a}}$ & 78.25 & $86.06^{\mathrm{ab}}$ & 58.15 & 1.60 \\
\hline T7: Neem cake@ 9500 kg ha ${ }^{-1}$ & $14.09^{b}$ & 56.28 & $9.07^{\mathrm{c}}$ & 34.04 & $73.97^{\mathrm{c}}$ & 51.30 & 1.54 \\
\hline T8: Phorate 10G@15kg ha ${ }^{-1}$ & $10.91^{\mathrm{b}}$ & 66.15 & $6.67^{\mathrm{b}}$ & 51.49 & $65.67^{\mathrm{d}}$ & 45.14 & 1.38 \\
\hline $\begin{array}{l}\text { T9: Untreated control only } 250 \mathrm{~kg} \\
\text { FYM }\end{array}$ & $32.23^{\mathrm{c}}$ & - & $13.75^{\mathrm{d}}$ & - & $36.02^{\mathrm{e}}$ & - & 0.78 \\
\hline $\mathrm{CD}(\mathrm{P}=0.05)$ & 7.6 & & 1.78 & & 4.53 & & \\
\hline CV\% & 20.56 & & 20.88 & & 3.35 & & \\
\hline
\end{tabular}

Values in column followed by different letter $(a, b, c)$ are significantly different from each other 
RAMANUJAM et al.

Table 4. Effect of bioagents on non-target organisms in sugarcane ecosystem during 2015-16

\begin{tabular}{|c|c|c|c|c|c|c|c|c|c|c|c|c|c|c|c|}
\hline \multirow[t]{3}{*}{ Treatments } & \multicolumn{15}{|c|}{ *Mean number/3 plants or places } \\
\hline & \multicolumn{3}{|c|}{$\begin{array}{c}\text { Earthworm } \\
\text { (No./m3) }\end{array}$} & \multicolumn{3}{|c|}{$\begin{array}{c}\text { Earwig Population } \\
\text { (No./ m3) }\end{array}$} & \multicolumn{3}{|c|}{ Spiders } & \multicolumn{3}{|c|}{ Coccinellids } & \multicolumn{3}{|c|}{ Chrysopids } \\
\hline & $\begin{array}{c}1 \\
\text { DBT }\end{array}$ & $\begin{array}{c}30 \\
\text { DAT }\end{array}$ & $\begin{array}{c}60 \\
\text { DAT }\end{array}$ & $\begin{array}{c}1 \\
\text { DBT }\end{array}$ & $\begin{array}{c}30 \\
\text { DAT }\end{array}$ & $\begin{array}{c}60 \\
\text { DAT }\end{array}$ & $\begin{array}{c}1 \\
\text { DBT }\end{array}$ & $\begin{array}{c}30 \\
\text { DAT }\end{array}$ & $\begin{array}{c}60 \\
\text { DAT }\end{array}$ & $\begin{array}{c}1 \\
\text { DBT }\end{array}$ & $\begin{array}{c}30 \\
\text { DAT }\end{array}$ & $\begin{array}{c}60 \\
\text { DAT }\end{array}$ & $\begin{array}{c}1 \\
\text { DBT }\end{array}$ & $\begin{array}{c}30 \\
\text { DAT }\end{array}$ & $\begin{array}{c}60 \\
\text { DAT }\end{array}$ \\
\hline $\begin{array}{l}\mathrm{T} 1 \text { : Metarhizium an- } \\
\text { isopliae } \\
\text { (NBAIR Ma4) } 2.0 \% \text { W.P } \\
@ 2.5 \mathrm{~kg} \mathrm{ha}^{-1} 1 \times 10^{8} \mathrm{Cfu} / \\
\text { gm (min.) in } 250 \mathrm{~kg} \\
\text { FYM (2 times) }\end{array}$ & 1.0 & 2.00 & 2.33 & 0.00 & 0.33 & 0.67 & 0.67 & 0.67 & 1.0 & 2.0 & 1.67 & 1.33 & 2.33 & 2.67 & 1.33 \\
\hline $\begin{array}{l}\text { T2: Beauveria bassiana } \\
\text { (NBAIR Bb5a) } 2.0 \% \\
\text { W.P @ } 2.5 \mathrm{~kg} \text { ha } \\
{ }^{-} 1 \times 10^{8} \mathrm{Cfu} / \mathrm{g} \text { in } 250 \mathrm{~kg} \\
\mathrm{FYM}(2 \text { times })\end{array}$ & 0.67 & 1.33 & 2.0 & 0.33 & 0.33 & 0.00 & 1.33 & 1.67 & 1.00 & 1.67 & 1.33 & 1.33 & 3.00 & 2.67 & 2.33 \\
\hline $\begin{array}{l}\text { T3: Heterorhabditis in- } \\
\text { dica (NBAIR H38) WP } \\
\text { @ } 20 \mathrm{~kg} \text { ha- } 1 \text { in } 150 \mathrm{~kg} \\
\text { moist sand ha }{ }^{-1}\end{array}$ & 0.67 & 1.33 & 1.67 & 0.33 & 0.67 & 0.33 & 0.67 & 0.33 & 0.33 & 1.67 & 1.33 & 1.00 & 2.67 & 2.67 & 2.00 \\
\hline $\begin{array}{l}\text { T4: Heterorhabditis in- } \\
\text { dica (NBAIR H38) WP } \\
\text { @ } 20 \mathrm{~kg} \mathrm{ha}^{-1} \text { in } 150 \mathrm{~kg} \\
\text { moist sand ha }{ }^{-1} \text { two } \\
\text { times at two month } \\
\text { interval }\end{array}$ & 1.00 & 1.00 & 1.33 & 0.00 & 0.33 & 0.00 & 0.00 & 0.33 & 1.00 & 1.33 & 1.00 & 0.67 & 2.00 & 1.33 & 0.67 \\
\hline $\begin{array}{l}\text { T5: Steinernema car- } \\
\text { pocapsae (NBAII Sc05) } \\
\text { WP @ } 20 \mathrm{~kg} \mathrm{ha}^{-1} \text { in } 150 \\
\text { kg moist sand ha }{ }^{-1}\end{array}$ & 1.00 & 0.67 & 1.67 & 0.00 & 0.33 & 0.00 & 0.67 & 0.33 & 1.00 & 2.33 & 1.33 & 1.33 & 2.67 & 2.33 & 1.00 \\
\hline $\begin{array}{l}\text { T6: Steinernema car- } \\
\text { pocapsae (NBAII Sc05) } \\
\text { WP @ } 20 \mathrm{~kg} \mathrm{ha}^{-1} \text { in } \\
150 \mathrm{~kg} \text { moist sand ha-1 } \\
\text { two times at two month } \\
\text { interval }\end{array}$ & 1.00 & 1.33 & 1.67 & 0.67 & 0.00 & 0.00 & 1.00 & 0.67 & 0.67 & 0.67 & 1.00 & 1.33 & 2.00 & 2.00 & 1.33 \\
\hline $\begin{array}{l}\text { T7: Neem cake @ } 500 \\
\text { kg ha }^{-1}\end{array}$ & 0.67 & 1.00 & 1.33 & 0.00 & 0.00 & 0.00 & 1.67 & 0.33 & 0.67 & 1.00 & 1.00 & 1.0 & 2.33 & 2.33 & 1.00 \\
\hline $\begin{array}{l}\text { T8: Phorate 10G@15kg } \\
\text { ha }^{-1}\end{array}$ & 1.00 & 0.67 & 0.0 & 0.00 & 0.00 & 0.00 & 0.67 & 1.00 & 0.67 & 1.00 & 1.00 & 0.67 & 2.33 & 1.33 & 1.33 \\
\hline $\begin{array}{l}\text { T9: Untreated controlon- } \\
\text { ly } 250 \mathrm{~kg} \text { FYM }\end{array}$ & 0.67 & 0.00 & 0.00 & 0.00 & 0.00 & 0.00 & 0.67 & 0.67 & 0.67 & 1.33 & 1.33 & 1.00 & 1.67 & 1.67 & 1.67 \\
\hline $\mathrm{CD}(0.05)$ & NS & NS & NS & NS & NS & NS & NS & NS & NS & NS & NS & NS & NS & NS & NS \\
\hline
\end{tabular}


Management of white grub, Holotrichia consanguinea using biocontrol agents in sugarcane

Table 5. Effect of bioagents on non target organisms in sugarcane ecosystem during 2016-17

\begin{tabular}{|c|c|c|c|c|c|c|c|c|c|c|c|c|c|c|c|}
\hline \multirow[t]{3}{*}{ Treatments } & \multicolumn{15}{|c|}{ *Mean number/3 plants or places } \\
\hline & \multicolumn{3}{|c|}{$\begin{array}{l}\text { Earthworm } \\
(\text { No./m3 ) }\end{array}$} & \multicolumn{3}{|c|}{$\begin{array}{l}\text { Earwig Population } \\
\text { (No./ m3) }\end{array}$} & \multicolumn{3}{|c|}{ Spiders } & \multicolumn{3}{|c|}{ Coccinellids } & \multicolumn{3}{|c|}{ Chrysopids } \\
\hline & $\begin{array}{l}1 \\
\text { DBT }\end{array}$ & $\begin{array}{l}30 \\
\text { DAT }\end{array}$ & $\begin{array}{l}60 \\
\text { DAT }\end{array}$ & $\begin{array}{l}1 \\
\text { DBT }\end{array}$ & $\begin{array}{l}30 \\
\text { DAT }\end{array}$ & $\begin{array}{l}60 \\
\text { DAT }\end{array}$ & $\begin{array}{l}1 \\
\text { DBT }\end{array}$ & $\begin{array}{l}30 \\
\text { DAT }\end{array}$ & $\begin{array}{l}60 \\
\text { DAT }\end{array}$ & $\begin{array}{l}1 \\
\text { DBT }\end{array}$ & $\begin{array}{l}30 \\
\text { DAT }\end{array}$ & $\begin{array}{l}60 \\
\text { DAT }\end{array}$ & $\begin{array}{l}1 \\
\text { DBT }\end{array}$ & $\begin{array}{l}30 \\
\text { DAT }\end{array}$ & $\begin{array}{l}60 \\
\text { DAT }\end{array}$ \\
\hline $\begin{array}{l}\text { T1: Metarhizium } \\
\text { anisopliae } \\
\text { (NBAIR Ma4) } \\
2.0 \% \text { W.P @ } 2.5 \mathrm{~kg} \\
\mathrm{ha}^{-1} 1 \times 10^{8} \mathrm{Cfu} / \mathrm{gm} \\
\text { (min.) in } 250 \mathrm{~kg} \\
\text { FYM (2 times) }\end{array}$ & 0.00 & 1.67 & 2.00 & 0.33 & 2.00 & 0.67 & 1.00 & 1.00 & 2.00 & 1.00 & 1.00 & 1.67 & 2.33 & 1.67 & 2.67 \\
\hline $\begin{array}{l}\text { T2: Beauveria } \\
\text { bassiana } \text { (NBAIR } \\
\text { Bb5a) } 2.0 \% \\
\text { W.P@ } 2.5 \mathrm{~kg} \mathrm{ha} \\
{ }^{1} 1 \times 10^{8} \mathrm{Cfu} / \mathrm{g} \text { in } 250 \\
\mathrm{~kg} \mathrm{FYM}(2 \text { times) }\end{array}$ & 0.00 & 1.00 & 1.00 & 0.00 & 1.67 & 1.00 & 1.00 & 1.67 & 1.67 & 1.33 & 0.67 & 1.33 & 1.67 & 2.33 & 2.33 \\
\hline $\begin{array}{l}\text { T3: Heterorhabditis } \\
\text { indica (NBAIR } \\
\text { H38)WP @ } 20 \\
\text { kg ha-1 in } 150 \mathrm{~kg} \\
\text { moist sand ha-1 }\end{array}$ & 0.33 & 1.00 & 1.33 & 0.00 & 0.67 & 0.00 & 0.33 & 0.33 & 0.33 & 1.00 & 0.67 & 1.33 & 1.67 & 2.00 & 2.00 \\
\hline $\begin{array}{l}\text { T4: Heterorhabditis } \\
\text { indica (NBAIR } \\
\mathrm{H} 38) \text { WP @ } 20 \mathrm{~kg} \\
\text { ha }^{-1} \text { in } 150 \mathrm{~kg} \text { moist } \\
\text { sand ha }^{-1} \text { two } \\
\text { times at two month } \\
\text { interval }\end{array}$ & 0.00 & 1.00 & 0.67 & 0.67 & 0.00 & 0.00 & 0.33 & 0.33 & 1.00 & 0.67 & 0.33 & 1.00 & 1.33 & 1.67 & 1.33 \\
\hline $\begin{array}{l}\text { T5: Steinernema } \\
\text { carpocapsae } \\
\text { (NBAII Sc05) WP } \\
@ 20 \mathrm{~kg} \mathrm{ha}^{-1} \text { in } \\
150 \mathrm{~kg}^{2} \text { moist sand } \\
\mathrm{ha}^{-1}\end{array}$ & 0.67 & 0.67 & 2.33 & 0.00 & 0.00 & 0.33 & 1.00 & 1.00 & 1.33 & 1.33 & 1.00 & 1.33 & 1.33 & 2.00 & 1.67 \\
\hline $\begin{array}{l}\text { T6: Steinernema } \\
\text { carpocapsae } \\
\text { (NBAII Sc05) WP } \\
\text { @ } 20 \mathrm{~kg} \mathrm{ha}^{-1} \text { in } 150 \\
\mathrm{~kg} \text { moist sand ha } \\
\text { two times at two } \\
\text { month interval }\end{array}$ & 0.00 & 1.33 & 0.00 & 0.00 & 0.00 & 0.00 & 0.67 & 1.00 & 0.67 & 1.33 & 0.67 & 1.00 & 1.67 & 1.33 & 2.00 \\
\hline $\begin{array}{l}\text { T7: Neem cake @ } \\
500 \mathrm{~kg} \mathrm{ha}^{-1}\end{array}$ & 0.67 & 1.00 & 0.67 & 0.00 & 0.33 & 0.67 & 0.67 & 1.00 & 0.33 & 1.00 & 0.33 & 1.00 & 1.67 & 1.00 & 2.00 \\
\hline $\begin{array}{l}\text { T8: Phorate 10G @ } \\
\text { 15kg ha }\end{array}$ & 1.00 & 0.67 & 0.00 & 0.00 & 0.00 & 0.00 & 0.67 & 1.00 & 0.33 & 0.67 & 0.33 & 1.00 & 1.33 & 1.67 & 1.67 \\
\hline $\begin{array}{l}\text { T9:Untreated } \\
\text { controlonly } 250 \mathrm{~kg} \\
\text { FYM }\end{array}$ & 0.67 & 0.00 & 0.00 & 0.00 & 0.00 & 0.00 & 0.67 & 0.67 & 0.67 & 1.00 & 0.67 & 1.33 & 1.33 & 1.33 & 0.67 \\
\hline $\mathrm{CD}(0.05)$ & NS & NS & NS & NS & NS & NS & NS & NS & NS & NS & NS & NS & NS & NS & NS \\
\hline
\end{tabular}


61.59 to $77.08 \mathrm{t} / \mathrm{ha}$. Untreated control recorded significantly very low cane yield of $24.93 \mathrm{t} /$ ha (Table 1 ).

In the second year trial (2016-17), the cane yield was significantly high in T4 $H$. indica (NBAIR H38) (twice application) treatment (101t/ha), which was statistically at par with other treatments like, T3, H indica (NBAIR H38) (single application) (99.8t/ha), T6 and T5: S. carpocapae (NBAII Sc05) (twice and single application) with an yield of 98.7 and 98.49 t/ha T1,M. anisopliae treatment (97.73t/ha) and T2, B. bassiana $(92.82 \mathrm{t} / \mathrm{ha})$ (Table 2). Other treatments like, Phorate 10G/Neem cake application/untreated control recorded significantly low cane yields of 69.74, 86.02 and $47.12 \mathrm{t} /$ ha respectively (Table 2 ).

The pooled data analysis of two years indicates that all biocontrol agent treatments (T1, T2, T3, T4, T5 and T6 treatments were at par with each other with regard cane yield (83.9-92.58t/ha), although T1 (M. anisopliae NBAIR Ma4) treatment showed highest yield of $92.58 \mathrm{t} /$ ha (Table 3 ).

\section{Cost Benefit Ratio}

The cost benefit ratio (2.0) was found highest in $M$. anisopliae (NBAIR Ma4) 2.0\% W.P. formulation treatment in both years (2.0 and 2.03) which was followed by B. bassiana (NBAIR Bb5a) treatment (1.71 and 1.93) and rest of the treatments showed $\mathrm{C}: \mathrm{B}$ ratio in the range of $0.57-1.50$ (2015) and 0.98-1.82 (2016-17) (Table 1 and 2).

\section{Effect of non target organisms}

Statistically significant differences were not observed in the population levels of non-target organisms like soil beneficial organisms like, Earthworms and Earwigs and predators like, Spiders, Coccinellids and Chrysopids in all treated plots and in the untreated control plots in both years (2015 and 2016-17) (Table 3 and 4).

The pooled data analysis of two years indicates that all biocontrol agent treatments were at par with each other with regard to percent plants damaged by white grubs and grub population. Regarding yield, treatments with $M$. anisopliae, $H$. indica (single and twice application) and S. carpocapsae (twice application) showed significantly higher yields compared to the yields in B. Bassiana and S. carpocapsae (single application). However, when cost benefit ratio is taken in to consideration, treatment with $M$. anisopliae proved superior to the other biocontrol agent treatments. Other treatments like, Phorate 10G/Neem cake application/ untreated control recorded significantly high percent of plant damage, grub population and low yield compared the treatments with biocontrol agents. Significant differences were not observed in the population levels of non-target organisms like earthworms, earwigs, spiders, coccinellids and chrysopids in all treatments and in untreated control plots indicating their non-deleterious effect on the beneficial soil organisms and natural enemies of insect pests.

Kulye and Pokharkar (2009) reported the efficacy of soil application of $M$. anisopliae@ $2 \times 10^{12}$ conidia ha $^{-1}$ $46.74 \%$ reduction of grub population of $H$. consanguinea and increased yield of $27.6 \mathrm{t} \mathrm{ha}^{-1}$ in potato. Application of $M$. anisopliae at the dose of $4 \times 10^{9}$ conidia ha-1against sugarcane white grub Holotrichia serrata (Blanch) was found effective with $92 \%$ reduction of grub population and increased yield of $100.6 \mathrm{t} \mathrm{ha}^{-1}$ and was found next to chlorpyriphos treatment (Manisegaran et al., 2011). Samson et al. (1999) showed that, soil application of M. anisopliae@3.3 x 10 13 conidia ha-1 against gray back cane grub (Dermolepida albohirtum) in Australia showed 50-60 per cent reduction in grub population in sugarcane.M. anisopliae and B.bassiana@5x10 $10^{8}$ conidia ha $^{-1}$ along with chlorpyriphos @ 2lit. ha ${ }^{-1}$ was found effective in reducing grub population (Bhagat et al., 2003). Kumbhar et al. (2019) reported superior field efficacy of $M$. anisopliae-talc based at the dose of $5 \mathrm{gm}$ per litre against the white grub, Leucopholis lepidophora in sugarcane in Maharashtra with minimal clump mortality (9.36\%) followed by the application of entomopathogenic Nematode-Powder with the dose of $5 \mathrm{gm}$ per litre (11.68\% clump mortality). In Germany, natural epizootics of Heterorhabditis sp was reported in grub populations achieving 71\% control of the pest in sugarcane field (Akhurst et al., 1992). In our study, soil application of biocontrol agents during two-year field trials showed $68-95 \%$ reduction of pest population and 45 $62 \%$ increased yield of over control in sugarcane crop in coastal Andhra Pradesh. The present findings also indicated that all biocontrol agents tested showed no adverse effect on non-target organisms like earthworms, earwigs, spiders, Coccinellids and Chrysopids. Thungrabeab and Tongma (2007), reported safety of $B$. bassiana Bb.5335 and $M$. anisopliae Ma.7965 to non-target insects such as natural enemies viz., Coccinella septempunctata L., Chrysoperla carnea (Stephens) and Dicyphus tamaninii (Wagner) and beneficial soil insect Heteromurus nitidus (Templeton).

\section{CONCLUSION}

The results showed that soil application of biocontrol agents effectively reduced the grub population of $H$. consanguinea and increased yield in white grub infested sugarcane crop in Coastal Andhra Pradesh, India. Based on cost-benefit ratio, the treatment with $M$. anisopliae, (ICARNBAIR Ma-4) was found superior to all other treatments in the management of sugarcane white grubs in coastal Andhra Pradesh with no deleterious effect on non-target organisms. This treatment was better than the insecticidal application in the management of sugarcane white grubs. 


\section{ACKNOWLEDGEMENTS}

The authors are thankful to the Director, ICAR-National Bureau of Agricultural Insect Resources, Bengaluru, Karnataka, India and Director of Research, Associate Director of Agriculture, Regional Agricultural Research Station, ANGRAU, Anakapalli, Andhra Pradesh. India for the constant support provided throughout the research work.

\section{REFERENCES}

AkhurstRJ,BeddingRA,BullRM,SmithRJ.1992.Anepizootic of Heterorhabditis spp (Heterorhabditidae:Nematoda) in sugarcane scarabaeids (Coleoptera). Fundam Appl Nematol. 15: 71-73.

Kulye MS, Pokharkar DS. 2009. Evaluation of two species of entomopathogenic fungi against white grub, Holotrichia consanguinea (Blanchard) infesting potato in Maharashtra, India. J Biol Control. 23(1):1-4.

Kumar IN, Suneetha K. 2016. Profitability analysis and problems of sugarcane in Andhra Pradesh. Int J App Res. 2(8):77-80.

Kumbhar RA, Mohite PB, Baral SB. 2019. Bioefficacy of various formulations of biopesticides against white grub, Leucopholis lepidophora infesting sugarcane under field condition. Journal of Entomology and Zoology Studies. 7(5): 1041-1044.

Manisegaran S, Lakshmi SM, Srimohanapriya V. 2011. Field evaluation of Metarhizium anisopliae (Metschnikoff)
Sorokin against Holotrichia serrata (Blanch) in sugarcane. J Biopesticides. 4(2):190-193.

Samson PR, Milner RJ, Ballard GK, Hogorth DM. 1999. Development of Metarhizium as biopesticides for sugarcane pest management-current progress and future prospects. 156- $163 \mathrm{PP}$.

Selvi TC, Rhichard TW, Kandasamy R. 2010. Laboratory culture and virulence of Beauveria brongniartii isolates on sugarcane white grub, Holotrichia serrata F. $J$ Biopesticides. 3(1):177-179.

Sahayaray K, Borgio JF. 2009. Distribution of Metarhizium anisopliae (Metsch.) Sorokin (Deuteromycotina:Hyphomycetes) in Tamil Nadu, India, Its biocontrol potential on Dysdercus cingulatus (Fab.) (Hemiptera:Pyrrhocoridae). Arch Phytopathology Plant Protect. 42(5):424-435. https:// doi.org/10.1080/03235400601160065

Srikanth J, Singaravelu B. 2011. White grub (Holotrichia serrata) as pest of sugarcane and its management. Technical Bulletin No 197 pp.1-8. Sugarcane Breeding Institute, Coimbatore.

Thungrabeab M, Tongma S. 2007. Effect of enthomopatogenic fungi, Beauveria bassiana (Balsamo) and Metarhizium anisopliae (Metschnikoff) on non-target insect. J Techn. 7(1):8-12. 